\title{
MicroRNA-9 suppresses cell migration and invasion through downregulation of TM4SF1 in colorectal cancer
}

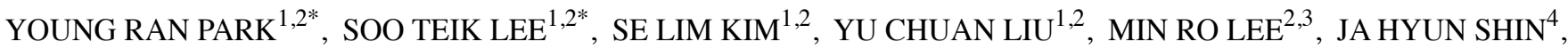 \\ SEUNG YOUNG SEO ${ }^{1,2}$, SEONG HUN KIM ${ }^{1,2}$, IN HEE KIM ${ }^{1,2}$, SEUNG OK LEE ${ }^{1,2}$ and SANG WOOK KIM ${ }^{1,2}$ \\ ${ }^{1}$ Department of Internal Medicine, ${ }^{2}$ Research Institute of Clinical Medicine of Chonbuk National University-Biomedical \\ Research Institute, ${ }^{3}$ Department of Surgery, Chonbuk National University Hospital, Chonbuk National University Medical \\ School, Jeonju; ${ }^{4}$ Department of Nursing Science, Vision University College of Jeonju, Jeonju, Republic of Korea
}

Received January 12, 2016; Accepted February 6, 2016

DOI: $10.3892 /$ ijo.2016.3430

\begin{abstract}
Transmembrane-4-L6 family 1 (TM4SF1) is upregulated in colorectal carcinoma (CRC). However, the mechanism leading to inhibition of the TM4SF1 is not known. In the present study, we investigated the regulation of TM4SF1 and function of microRNAs (miRNAs) in CRC invasion and metastasis. We analyzed 60 colon cancers and paired normal specimens for TM4SF1 and miRNA-9 (miR-9) expression using quantitative real-time PCR. A bioinformatics analysis identified a putative miR-9 binding site within the 3'-UTR of TM4SF1. We also found that TM4SF1 was upregulated in CRC tissues and CRC cell lines. The expression of TM4SF1 was positively correlated with clinical advanced stage and lymph node metastasis. Moreover, a luciferase assay revealed that miR-9 directly targeted 3'-UTR-TM4SF1. Overexpression of miR-9 inhibited expression of TM4SF1 mRNA and protein, wound healing, Transwell migration and invasion of SW480 cells, whereas, overexpression of anti-miR-9 and siRNATM4SF1 inversely regulated the TM4SF1 mRNA and protein level in HCT116 cells. Furthermore, miR-9 suppressed not only TM4SF1 expression but also MMP-2, MMP-9 and VEGF expression. In clinical specimens, miR-9 was generally downregulated in CRC and inversely correlated with TM4SF1 expression. These results suggest that miR-9 functions as a tumor-suppressor in CRC, and that its suppressive effects mediate invasion and metastasis by inhibition of TM4SF1 expression. Our results also indicate that miR-9 might be a novel target for the treatment of CRC invasion and metastasis.
\end{abstract}

Correspondence to: Dr Sang Wook Kim, Department of Internal Medicine, Research Institute of Clinical Medicine, Biomedical Research Institute, Chonbuk National University Hospital, 20 Geonji-ro, Deokjin-gu, Jeonju, Jeonbuk 561-712, Republic of Korea E-mail: clickm@jbnu.ac.kr

*Contributed equally

Key words: colorectal cancer, transmembrane-4-L6 family 1, miR-9, invasion, metastasis

\section{Introduction}

Colorectal cancer (CRC) is the third most common malignant disease in the world (1). Despite many advances in medicine, nearly $50 \%$ of CRC patients show tumor recurrence, which is leading to poor prognosis, median survival ratio following recurrence is only 13.3 months (2). Most recurrences of CRC are thought to be the result of tumor invasion and metastasis of cancer cells. Therefore, understanding the molecular mechanisms in CRC metastasis is of crucial significance in developing therapeutic strategies to improve CRC patients.

Transmembrane-4-L6-family-1 (TM4SF1) is a 22-kDa four-transmembrane-domain protein. It was identified in 1986 as a tumor cell antigen of mouse monoclonal antibody L6, which also has a low expression in normal vascular endothelium $(3,4)$. There are five other structurally similar proteins: TM4SF4/IL-TMP, TM4SF5/L6H, TM4SF18/L6D, TM4SF19/ OCTM4 and TM4SF20/TCCE518 (5). Recently, studies have shown that TM4SF1 is associated with tumor growth, motility, invasion and metastasis with high expression in human lung, breast, colon, ovarian, renal and prostate carcinomas (3,6-10). In particular, TM4SF1 has high expression in CRC tissues, and downregulation of TM4SF1 can decrease the progression and metastasis of CRC (11). Therefore, TM4SF1 inhibition might provide a strategy for treating CRC.

MicroRNAs (miRNAs) are a new classification of endogenous, small, single-stranded RNAs composed of 19-24 nucleotides. miRNAs modulate gene expression by binding to the 3'-untranslated region (3'-UTR) of the target mRNA, resulting in downregulation of the mRNA transcript or inhibition of the protein translation process (12). In addition, miRNAs regulate many cellular processes, including apoptosis, cell cycle progression, proliferation, differentiation, invasion and migration and affect tumorigenesis (13-19). Thus, understanding the underlying molecular mechanisms of miRNA in malignant tumors is critical to CRC therapy. In this study, we focused on miRNA-9 (miR-9) because downregulation of its expression has been observed in several cancer types, such as cervical adenocarcinoma, breast, gastric, ovarian and hepatocellular carcinoma (20-24). In CRC, miR-9 expression is also downregulation by binding to the 3 '-UTR, and it has the potential to suppress Cdx2 (caudal-type homeobox 2) and 
UHRF1 (ubiquitin-like with plant homeodomain and ring finger domain 1), leading to proliferation, apoptosis, migration and invasion (25-27). However, little is known about the factors that modulate TM4SF1 in CRC invasion and metastasis. We hypothesized that miRNAs are associated with TM4SF1 expression in CRC motility.

We analyzed the expression of TM4SF1 in 60 paired CRC tissues and found that the expression level of TM4SF1 was significantly higher in CRC tumors than in normal tissues, and the expression level of TM4SF1 was associated with clinical pathological stage and lymph node metastasis. Moreover, miR-9 directly targeted its binding site in the TM4SF1 3'-UTR, which has a critical role in regulating CRC cell migration and invasion. Furthermore, miR-9 regulated cell motility via suppressing MMP-2, MMP-9 and VEGF expression in CRC cell lines. Taken together, miR-9 is associated with the motility of CRC and can be used for molecular targeted therapies in CRC.

\section{Materials and methods}

Cell culture. Human colorectal cancer cell lines SW480, Caco2, LS174T, SW620 and HCT116 were purchased from the American Type Culture Collection (ATCC; Manassas, VA, USA). The cells were grown in RPMI-1640 (Invitrogen, Carlsbad, CA, USA) according to the manufacturer's recommendations, with $10 \%$ fetal bovine serum (FBS) and $1 \%$ streptomycin at a humidified $5 \% \mathrm{CO}_{2}$ at $37^{\circ} \mathrm{C}$.

MicroRNAs transfection and siRNA treatment. Both miR-9 (hsa-miR-9a-5p; Pre-miRNA, miRNA Precursor AM17100; Product ID: PM 10022) and anti-miR-9 (anti-hsa-miR-9a-5p; anti-miRNA, miRNA inhibitor AM17100; Product ID: AM10022) were commercially synthesized (Ambion, Austin, TX, USA). The miR control and anti-miR control were purchased from Shanghai GenePharma Co., Ltd., (Shanghai, China) and siRNA-TM4SF1 was commercially synthesized (Thermo Fisher Scientific, Rockford, IL, USA). SW480 and HCT116 cells $\left(2-5 \times 10^{5}\right)$ were plated in 6-well plates and cultured for one day before transfection. For transfection, miRNAs or siRNA were used at working concentrations of 50 or $20 \mathrm{nM}$ using Lipofectamine 2000 reagent (Invitrogen). Cells were harvested at 24, 48 and $72 \mathrm{~h}$ after transfection for miRNA, mRNA and protein, respectively.

Patients and tissue specimens. Sixty of CRC tissues and paired normal tissues were obtained through the Biobank of Chonbuk National University Hospital, a member of the National Biobank of Korea. All patients had a pathological diagnosis of CRC, each paired sample was classified according to TNM Classification of Malignant Tumours (TNM) classification and were frozen in liquid nitrogen and stored at $-80^{\circ} \mathrm{C}$. The characteristics of patients are shown in Table I. This study consisted of 25 (41.7\%) females and 35 (58.3\%) males with a mean age of 63.1 years. The study protocol was approved by the Institutional Review Boards of Chonbuk National University Hospital (IRB no. 2014-10-05).

RNA isolation and real-time quantitative polymerase chain reaction (RTQ-PCR) for quantification of miR-9 and TM4SF1.
Table I. Characteristics of the $60 \mathrm{CRC}$ patients.

\begin{tabular}{lr}
\hline Variables & $\mathrm{N}(\%)$ \\
\hline Gender & \\
Male & $35(58.3)$ \\
Female & $25(41.7)$ \\
Age (years) & \\
$<60$ & $24(40.0)$ \\
$\geq 60$ & $36(60.0)$ \\
Histological differentiation & \\
Well & $8(13.3)$ \\
Moderate & $46(76.7)$ \\
Poor & $6(10.0)$
\end{tabular}

Tumor status (T)

T1-T2

$20(33.3)$

T3-T4

$40(66.7)$

Lymph node metastasis $(\mathrm{N})$

NO

$33(55.0)$

N1-N2

14 (23.3)

N3-N4

$13(21.7)$

Distant metastasis status (M)

M0

$57(95.0)$

M1

AJCC

I + II

$30(50.0)$

III + IV

$30(50.0)$

AJCC, American Joint Committee on Cancer.

Total RNA from cells or human normal tissue/matched tumor samples was extracted using TRIzol reagent (Invitrogen, Carlsbad, CA, USA). Reverse transcription was performed using M-MLV Reverse Transcriptase (Promega, Madison, WI, USA), according to the manufacturer's protocol. RTQ-PCR was performed using an ABI 7500 real-time PCR system (Applied Biosystems, Foster City, CA, USA). In brief, $20 \mu \mathrm{l}$ of Master Mix was prepared on ice with $10 \mu \mathrm{l}$ of $2 \mathrm{X}$ SYBR, $1 \mu 1$ of primers, $2 \mu \mathrm{l}$ of DNA and $7 \mu \mathrm{l}$ of nuclease-free water. The Master Mix was initially denatured $95^{\circ} \mathrm{C}$ for $10 \mathrm{~min}$ followed by 40 cycels of denaturation at $95^{\circ} \mathrm{C}$ for $15 \mathrm{sec}$, annealing and extension at $60^{\circ} \mathrm{C}$ for $30 \mathrm{sec}$. The geometric average $\mathrm{Ct}$ value was used to calculate relative expression of the TM4SF1 using the method $2^{-\Delta \Delta \mathrm{CT}}$, which was normalized to beta-2-microglobulin (B2M). Primers used in this experiment were: 5'-TCG CGGCTAATATTTTGCTT-3' (forward) and 5'-TGCAATT CCAATGAGAGCAG-3' (reverse) for TM4SF1; and 5'-CCTG AATTGCTATGTGTCTGGG-3' (forward) and 5'-TGATG CTGCTTACATGTCTCGA-3' (reverse) for B2M. Expression level of miR-9 was determined by the TaqMan miRNA assay kit (Applied Biosystems) and normalized using the RNU48. The reaction volume of $20 \mu \mathrm{l}$ included 2X Master Mix, each primer $2 \mu \mathrm{l}$, cDNA $2 \mu \mathrm{l}$, nuclease-free water $6 \mu \mathrm{l}$, and amplification was carried out as follows: $95^{\circ} \mathrm{C}$ for $10 \mathrm{~min}, 40$ cycles of 
$95^{\circ} \mathrm{C}$ for $30 \mathrm{sec}, 60^{\circ} \mathrm{C}$ for $1 \mathrm{~min}$ and all the samples were performed in triplicate.

TM4SF1 target prediction by bioinformatics methods. To predict the target miRNAs of TM4SF1, we used bioinformatics software, TargetScan (www.targetscan.org), PicTar (www. mdc-berlin.de), Pita and miRanda-mirSVR (www.microrna. org), and combined with literature (27-30), miR-9 was selected for further study.

Plasmid construction and reporter assays. TM4SF1 plasmid DNA was kindly donated by Dr R. Roffler (Academia Sinica, Taipei, Taiwan) and was used to generate a new construct containing the full open reading frame (ORF) of the TM4SF1 gene (pcDNA3.1-TM4SF1). The wild-type and mutant TM4SF1 containing the predicted binding site for miR-9 were amplified by PCR using the primers: 5'-CTCGAGCCCTT TGAACTGCCTTGTGT-3' (forward) and 5'-CTCGAGCC CAGTCATCGTAGCCTTTC-3' (reverse) for TM4SF1-WT: 5'-GGAAAGCCTTTTGTCCTTGAGTACTAGGGATCAT G-3' (forward) and 5'-CATGATCCCTAGTACTCAAGGACA AAAGGCTTTC-3' (reverse) for TM4SF1-MT. The PCR product was cloned into the pmirGLO Dual-Luciferase miRNA target expression vector (Promega), designated TM4SF1-WT after sequencing. TM4SF1-MT was carried out using a site-directed mutagenesis kit (Enzynomics, Daejeon, Korea), using TM4SF1-WT as a template.

For the reporter assay, $5 \times 10^{4}$ of SW480 cells were seeded in a 24-well plate and transiently transfected with $200 \mathrm{ng}$ of TM4SF1-WT or TM4SF1-MT reporter plasmid with $50 \mathrm{nM}$ of miR-9 or miR negative control using Lipofectamine 2000. Luciferase assays were performed at $24 \mathrm{~h}$ after transfection using the Dual-luciferase assay system (Promega), and they were normalized with co-transfected Renilla luciferase. All experiments were performed in triplicate and repeated at least three times.

Western blot analysis. Protein extraction was prepared according to a previously described method (31). Briefly, cells were harvested by resolving in RIPA buffer $(50 \mathrm{mM}$ Tris- $\mathrm{HCl}$, $150 \mathrm{mM} \mathrm{NaCl}, 1 \%$ Triton $\mathrm{X}-100,1 \%$ sodium deoxycholate, $0.1 \% \mathrm{SDS}$ and protease inhibitors) and were centrifuged at $13,200 \mathrm{rpm}$ at $4^{\circ} \mathrm{C}$ for $30 \mathrm{~min}$. After centrifugation, supernatants were used as whole cell extracts, and 30-35 $\mu \mathrm{g}$ of protein was separated on 8 or $10 \%$ polyacrylamide gel and transferred to polyvinylidene difluoride (PVDF) membranes. The membranes were incubated with $2.5 \%$ non-fat dry milk or $2.5 \%$ BSA in TBST for $60 \mathrm{~min}$ and then incubated overnight at $4^{\circ} \mathrm{C}$ with primary antibodies to TM4SF1 (1:200; Thermo Fisher Scientific), MMP-2 (1:200; Cell Signaling Technology, Danvers, MA, USA), MMP-9 (1:200; Cell Signaling Technology) and VEGF (1:500; Santa Cruz Biotechnology, Santa Cruz, CA, USA). After washing three times with TBST, the membranes were incubated with HRP-conjugated rabbit or mouse IgG secondary antibodies for $60 \mathrm{~min}$ at room temperature. After washing three times with TBST, the proteins were visualized with ECL prime Western blotting substrate (Amersham, Buckinghamshire, UK) and detected with the chemiluminescent image system (Fusion Solo S; Vilber Lourmat, Marne-la-Valle'e Cedex, France). After protein detection, some membranes were re-probed with an antibody to GAPDH (Bioworld, Irving, TX, USA) used as a loading control.

Wound healing assay. Cells $\left(5 \times 10^{4}\right)$ were seeded in 24-well plates and incubated at $37^{\circ} \mathrm{C}$. The confluent cells were scratched with a $200-\mu 1$ pipette tip and then transfected as described above. After 24-h incubation, plates were washed with fresh medium to remove non-adherent cells and then photographed. Wound area was determined using an inverted microscope (IX71; Olympus, Center Valley, PA, USA).

Migration and invasion assay. Cell migration assay was performed using a Transwell system (24-wells, $8 \mu \mathrm{m}$ pore size with poly-carbonate membrane; SPL, Gyeonggi-do Korea) according to the manufacturer's instructions. Briefly, posttransfected cells were trypsinized, and $1 \times 10^{5}$ cells were seeded into the upper chamber with serum free opti-MEM media. The low chamber was filled with $800 \mu \mathrm{l}$ medium containing $10 \%$ FBS as a chemoattractant. After incubation for $48 \mathrm{~h}$, cells on the lower side of the filter were fixed in $3.8 \%$ formaldehyde for $20 \mathrm{~min}$ and stained with $0.1 \%$ crystal violet solution. The number of cells in five randomly selected fields was counted under a light microscope and analyzed statistically. For the invasion assay, the upper chamber was coated with extracellular matrix (BD Biosciences, Bedford, MA, USA), a soluble basement membrane matrix. The rest of the assay was performed as the migration assay.

Statistical analysis. Spearman's correlation analysis was used to determine the correlation between TM4SF1 and miR-9 expression. All of the data are shown as mean \pm standard deviation (SD). Statistical differences were analyzed using ANOVA and Student's t-test, and P-values $<0.05$ were considered statistically significant.

\section{Results}

TM4SF1 expression is elevated in CRC, and associated with tumor stage and lymph node metastasis. We first analyzed TM4SF1 expression in human colorectal cancer specimens, 60 frozen $\mathrm{CRC}$ tissues and paired normal colon tissues using RTQ-PCR. The mRNA level of TM4SF1 in CRC tissues were significantly higher than the mRNA level in paired normal tissues $(\mathrm{P}<0.001$ for all comparisons; Fig. 1A). We also found that high level of TM4SF1 expression was significantly associated with increasing stage of $\mathrm{CRC}(\mathrm{P}<0.05$ for all comparisons; Fig. 1B), suggesting that TM4SF1 has more important functions in late-stage than early-stage CRC. TM4SF1 expression also was significantly increased with lymph node metastasis compared with normal tissues $(\mathrm{P}<0.01$ and $\mathrm{P}<0.001$ for all comparisons; Fig. 1C). We analyzed the level of TM4SF1 protein in $6 \mathrm{CRC}$ tissues and found that TM4SF1 expression in 5 of 6 were higher than the level in the matched normal tissues (Fig. 1D). These results suggest that elevated TM4SF1 expression is associated with CRC metastasis.

miR-9 is a direct target for TM4SF1 in CRC and inversely correlated in 5 colorectal cancer cell lines. To identify miRNA 


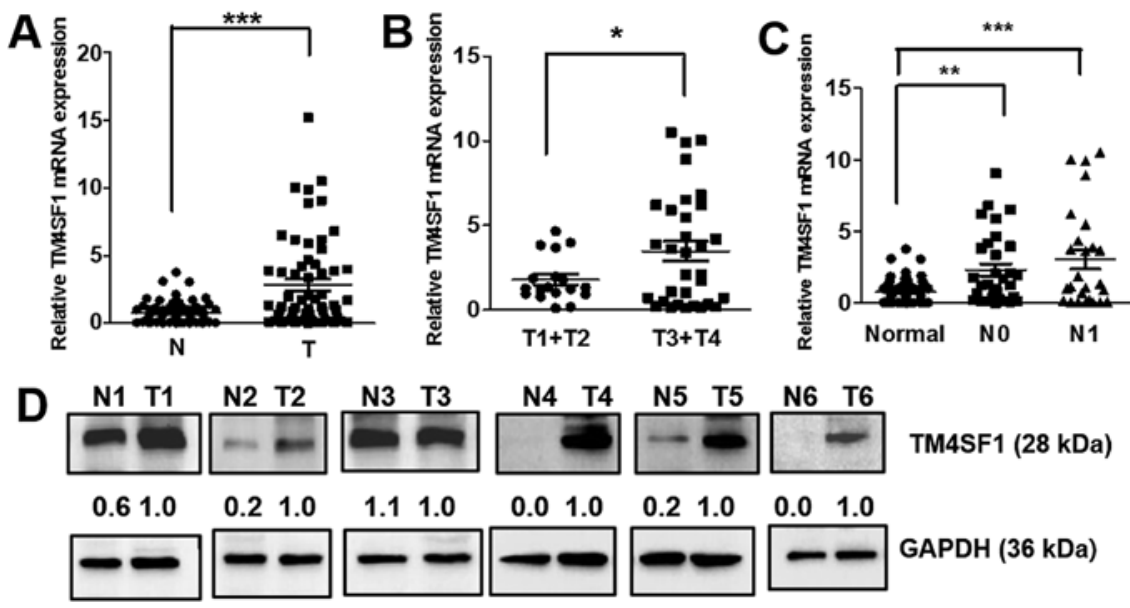

Figure 1. Expression of TM4SF1 is associated with increased stage and lymph node metastasis in 60 paired CRC specimens. (A) Expression levels of TM4SF1 in paired CRC tumor (T) and normal tissues (N). (B) Sixty CRC tumors tissues were divided according to the pathological classification stage (T1+T2) and stage (T3+T4). (C) Analysis of TM4SF1 expression in CRC tissues with or without lymph node metastases relative to match normal tissues. (D) Western blot analysis of TM4SF1 protein isolated from paired normal (N) and CRC tumor (T) tissue specimens. Tumor colon specimens display higher TM4SF1 protein levels. Data assessed from three independent experiments and the P-values were calculated by t-test $\left({ }^{*} \mathrm{P}<0.05,{ }^{* *} \mathrm{P}<0.01,{ }^{* * *} \mathrm{P}<0.0001\right)$.
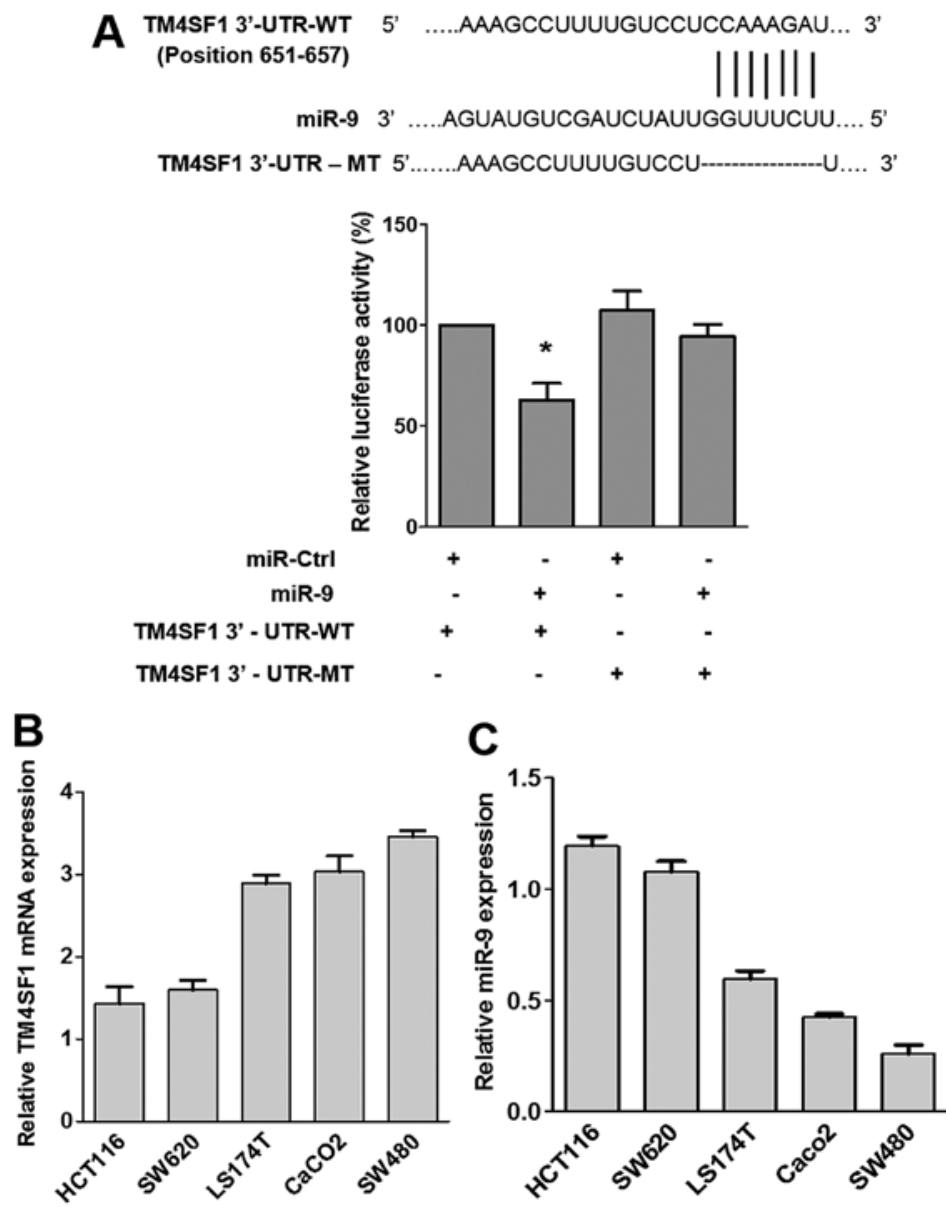

Figure 2. TM4SF1 is a direct target gene of miR-9 and miR-9 and TM4SF1 inversely correlate in 5 colorectal cancer cell lines (A) Sequence complementary between miR-9 and TM4SF1 3'-UTR. A human TM4SF1 3'-UTR containing the wild-type and mutant type miR-9 binding site was cloned into the pmirGLO Dual-Luciferase miRNA target expression vector. Luciferase activity was analyzed using the Dual-Luciferase assay system. (B) TM4SF1 mRNA expression was analyzed using RTQ-PCR in 5 CRC cell lines and normalized to B2M. (C) miR-9 expression were determined by TaqMan RTQ-PCR and normalized against an endogenous control (RNU48). Data assessed from three independent experiments and the P-values were calculated by t-test ("P<0.05).

target sites located in the $3^{\prime}$-UTR TM4SF1 mRNA, we used bioinformatics software, TargetScan and miRanda-mirSVR, and the results were combined and compared with findings in literature (27-30). miR-9 was found to be conserved in the binding site of TM4SF1 (Fig. 2A) and was therefore selected for further study. 

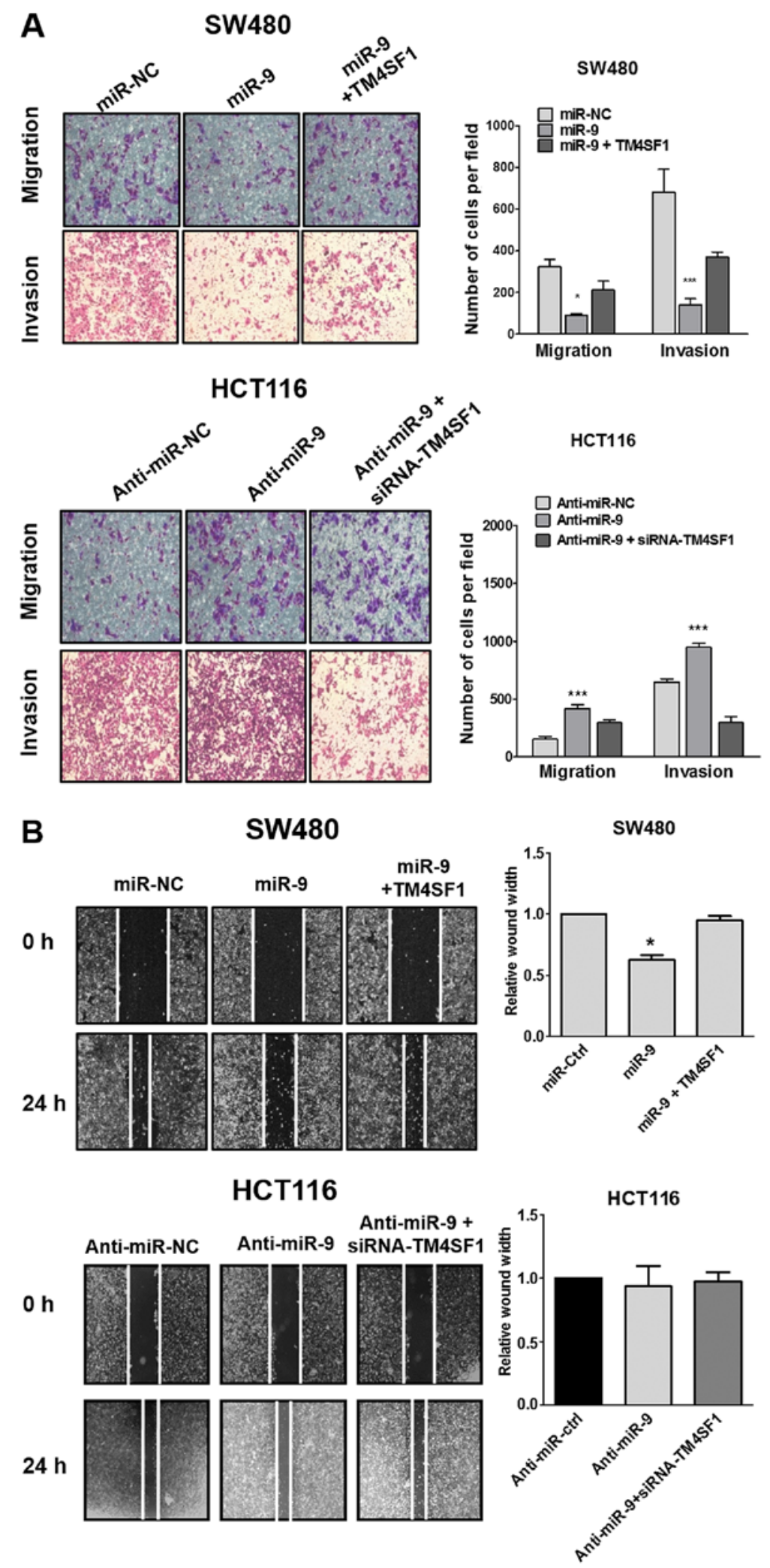

Figure 3. miR-9 downregulates cell migration and invasion in CRC cells. (A) Migration and invasion assay rescued the effects of TM4SF1 overexpression of inhibitory induction by miR-9 in SW480 cells. Overexpression of siRNA-TM4SF1 was downregulated in cell migration and invasion while upregulated the induction by anti-miR-9 in HCT116 cells. (B) Wound healing assay analysis of the rescued effects of TM4SF1 overexpression on inhibitory induction by miR-9 in SW480 cells. No significant change was observed on anti-miR-9 or co-transfection with anti-miR-9 and siRNA-TM4SF1 in HCT116 cells.

The luciferase assay showed that co-transfection of miR-9 and TM4SF1 3'-UTR-WT was significantly reduced by $\sim 40 \%$ compared with the co-transfection of the miR control and TM4SF1 3'-UTR-WT (Fig. 2A). However, no significant changes in luciferase activity were observed in co-transfection of either TM4SF1 3'-UTR-MT or the miR-control with miR-9 (Fig. 2A). These findings suggest that miR-9 directly targets TM4SF1 via the binding site in 3'-UTR region. 

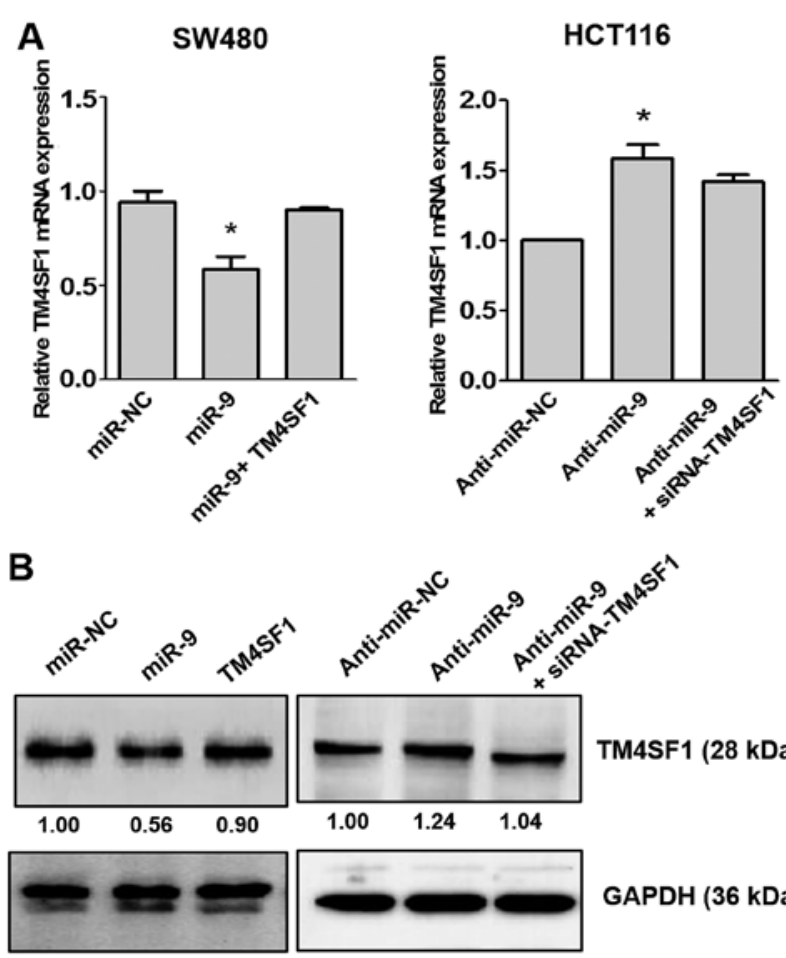

SW480

GAPDH (36 kDa)

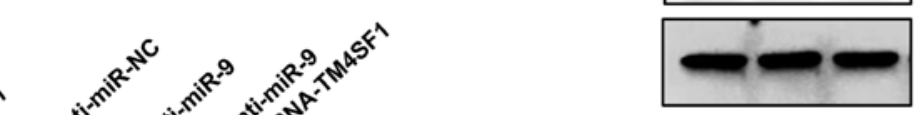

SW480
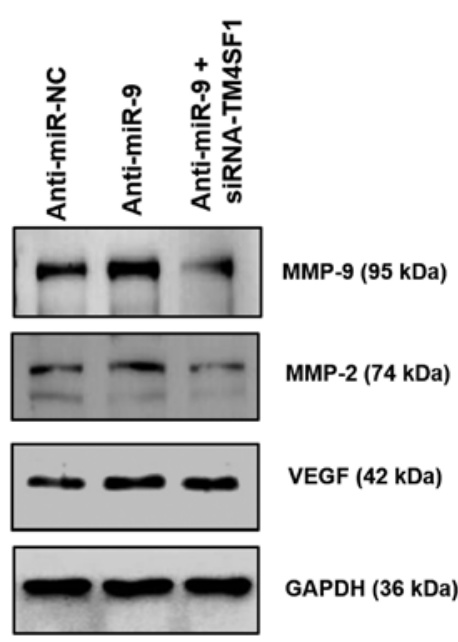

VEGF (42 kDa)

GAPDH (36 kDa)

Figure 5. miR-9 inhibits MMP-9/MMP-2/VEGF via targeting TM4SF1. MMP-9, MMP-2, VEGF expression rescued the effect of TM4SF1 overexpression on inhibitory induction by miR-9 in SW480 cells. Overexpression of anti-miR-9 and siRNA-TM4SF1 was downregulated is the protein expression, while upregulated induced by anti-miR-9 in HCT116 cells.

Figure 4. miR-9 downregulates TM4SF1 mRNA and protein level in CRC cells. (A) RTQ-PCR analysis of the rescued effect of TM4SF1 overexpression on inhibitory induction by miR-9 in SW480 cells $(\mathrm{P}<0.05)$ and TM4SF1 mRNA level was upregulated by overexpression of anti-miR-9, expression level recovered by cotransfection with anti-miR-9 and siRNA-TM4SF1 in HCT116 cells $(\mathrm{P}<0.05)$. (B) TM4SF1 overexpression rescues miR-9 mediated inhibition of TM4SF1 protein level in SW480 cells and anti-miR-9 enhanced siRNA-TM4SF1 mediated inhibition TM4SF1 protein level in HCT116 cells by western blot analysis. Data assessed from three independent experiments.

We also examined the expression levels of miR-9 and TM4SF1 in 5 colorectal cancer cell lines (HCT116, SW620, LS174T, Caco2 and SW480). As shown that Fig. 2B and C, the mRNA level of TM4SF1 was the highest in SW480 and the lowest in HCT116 cells. On the contrary, RTQ-PCR results showed that the expression of miR-9 was highest in HCT116 cells and lowest in SW480 cells. These findings suggest that the level of TM4SF1 expression is inversely correlated with miR-9 level in CRC cells. Based on these results, we used SW480 and HCT116 cells to analyze the gain or loss of TM4SF1.

miR-9 inhibits cell migration and invasion of CRC cells. To further evaluate the cellular effects of miR-9, miR-9 alone or miR-9 and TM4SF1 was transfected. MTT assay results showed that miR-9 significantly inhibited cell growth, but co-transfection of miR-9 or TM4SF1 had no effect on growth of SW480 and HCT116 cells (data not shown). To test the cell migration and invasion potential of CRC Transwell migration, invasion assay and wound healing in vitro were analyzed. As shown in Fig. 3, overexpression of miR-9 in SW480 cells significantly reduced cell migration $(\mathrm{P}<0.05)$ and invasion $(\mathrm{P}<0.01)$, co-transfection of miR-9 with TM4SF1 recovered these cellular functions. Whereas, overexpression of antimiR-9 in HCT116 cells significantly increased cell migration $(\mathrm{P}<0.001)$ and invasion compared with the control $(\mathrm{P}<0.001)$,

co-transfection of anti-miR-9 with siRNA-TM4SF1 also recovered these effects. Wound healing assay results showed that miR-9 overexpression significantly reduced the rate of wound healing $(\mathrm{P}<0.05)$, and co-transfection of miR-9 and TM4SF1 recovered the motility of SW480 cells, however, there was no significant effect of anti-miR-9 and siRNATM4SF1 in HCT116 cells (Fig. 3B). Collectively, miR-9 inhibits CRC cell migration and invasion in vitro by TM4SF1 expression.

miR-9 inhibits expression of TM4SF1 $m R N A$ and protein in $C R C$ cells. To test whether miR-9 regulates endogenous TM4SF1 expression, miR-9 and anti-miR-9 were transiently transfected into SW480 and HCT116 cells, and expression levels of TM4SF1 mRNA and protein were analyzed. As shown in Fig. 4A, transfection of miR-9 in SW480 cells lowered the TM4SF1 mRNA level compared with the level in the control $(\mathrm{P}<0.05)$, and co-transfection of miR-9 and TM4SF1 recovered expression of TM4SF1 mRNA. In contrast, the TM4SF1 mRNA level in HCT116 cells was enhanced by anti-miR-9 compared with the control $(\mathrm{P}<0.05)$, and the expression level was recovered by co-transfection with anti-miR-9 and siRNA-TM4SF1. Supporting the observations, that TM4SF1 protein expression was reduced by miR-9, and the expression level was also recovered by co-transfection of miR-9 and TM4SF1. In HCT116 cells, TM4SF1 expression was enhanced by anti-miR-9, and recovered by co-transfection of anti-miR-9 and siRNA-TM4SF1 (Fig. 4B). These results indicate that miR-9 regulates both the transcription and translation of TM4SFl.

Alteration of miR-9 expression influences MMP-9, MMP-2 and VEGF activation. To further understand the molecular mechanism of miR-9/TM4SF1 in inhibiting cell migration and invasion, we investigated whether it was due to the moderation of adhesion, which then lead to regulation of MMPs (matrix 

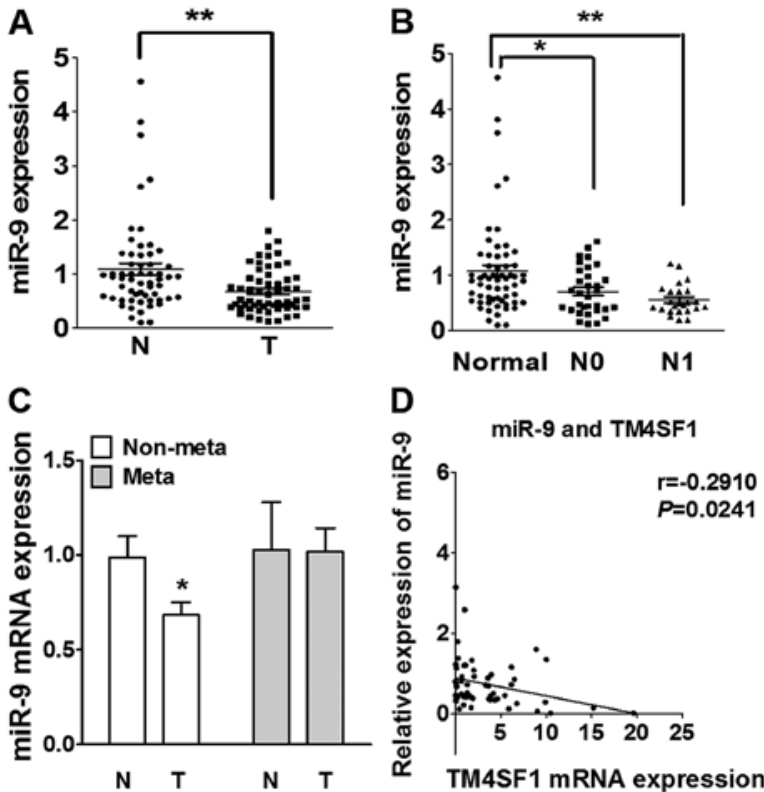

Figure 6. miR-9 was inversely correlated with expression of TM4SF1 in CRC specimens. The expression of miR-9 was analyzed in 60 paired normal and tumor tissues using RTQ-PCR and normalized to RNU48 as endogenous control. (A) miR-9 was frequently upregulated in normal tissues (N) compared with paired tumor specimens (T). (B) Analysis of miR-9 expression in CRC tissues with or without lymph node metastases relative to matched normal tissues. (C) miR-9 was significantly upregulated in normal nonmetastasis compared with tumor non-metastasis. (D) Spearman's correlation analysis was used to identify the correlation between the expression levels of TM4SF1 and miR-9 in human CRCs (Spearman's correlation $\mathrm{r}=-0.2910$, $\mathrm{P}=0.0241$ ). Data represent mean $\pm \mathrm{SD}$ of 3 replicates.

metalloproteinases) and the VEGF (vascular endothelial growth factor) signaling pathway $(32,33)$. Consequently, we analyzed expression of MMP-9, MMP-2, VEGF, ICAM-1 (intercellular adhesion molecule 1), VCAM-1 (vascular cell adhesion protein 1) in CRC cells.

Although miR-9 did not significantly change ICAM-1 or VACM-1 protein levels (data not shown), the expression of MMP-2, MMP-9 and VEGF was downregulated by miR-9 overexpression, this expression also recovered with co-transfection of miR-9 and TM4SF1 in SW480 cells (Fig. 5). In contrast, expression of TM4SF1 protein was upregulated by anti-miR-9, and this was recovered via co-transfection with anti-miR-9 and siRNA-TM4SF1 in HCT116 cells. Taken together, miR-9 not only downregulates the expression of TM4SF1, but also downregulates MMP-2, MMP-9 and VEGF expression in CRC cells.

TM4SF1 inversely correlates with miR-9 expression in CRC. To gain insight into the clinical implications of miR-9, expression of miR-9 was analyzed in 60 cases of human CRC using RTQ-PCR. The results revealed that expression level of miR-9 was significantly higher in normal tissues than in CRC tissues (P-value in each $<0.01$; Fig. 6A), in normal non-metastasis tissues than in tumor of non-metastasis ( $\mathrm{P}$-value in each $<0.05$; Fig. 6C). On the contrary, the level of miR-9 in CRC was significantly decreased with lymph node metastasis compared with normal tissues (P-value $<0.05$ and $<0.01$; Fig. 6B). We also evaluated that the correlation between TM4SF1 mRNA and miR-9 expression in the same CRC tissues. As shown in

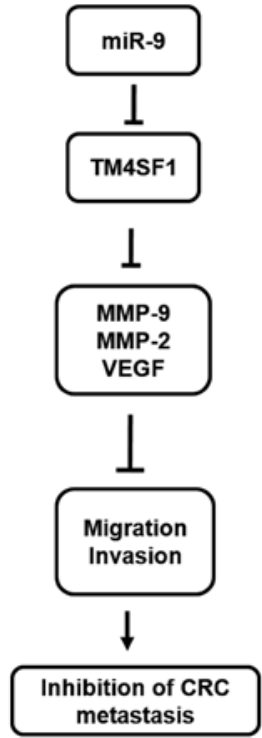

Figure 7. Diagram illustrating the possible molecular mechanisms through which miR-9 interacts with other molecules during the development of CRC invasion and metastasis.

Fig. 6D, Spearman's rank correlation analysis showed that the expression levels of miR-9 and TM4SF1 were inverse in the 60 CRC specimens.

\section{Discussion}

TM4SF1 is known to play important roles in growth, motility, invasion, and metastasis of cancer cells $(3,6-8)$. Previously, studies have identified aberrant expression of miRNAs in various types of cancer and analyzed the role of miRNAs in cancer development, process, metastasis and invasion $(34,35)$. In the present study, we focused on the expression of TM4SF1 in human CRC tissues and CRC cell lines along with the miRNA that regulates TM4SF1 expression. Our results showed that TM4SF1 is upregulated in 60 paired CRC specimens compared with corresponding normal tissues and is associated with increased stage and lymph node metastasis. The expression of TM4SF1 was significantly higher in late stages compared to early stages.

On the basis of the above findings, bioinformatics prediction analysis and literature search (27-30), were performed, miRNA-9 was selected as a potential target of TM4SF1. This prediction was confirmed by luciferase assay. Our results also revealed that miR-9 regulates TM4SF1 expression via the binding site in its 3'-UTR region thereby invasion and metastasis of CRC cells were reduced. These results suggest that miR-9 is strongly associated with CRC cell motility via its direct target gene the TM4SF1.

Clinically, increased expression levels of MMP-2 and MMP-9 in tumors are significantly associated with metastatic potential (36-38). Overexpression of MMP-2 and MMP-9 was also identified as prognostic markers of CRC (39). Our results show that miR-9 regulated the protein level of MMP-2/ MMP-9 and VEGF via TM4SF1 in CRC cells. A similar study of VEGF expression found that loss of TM4SF1 inhibited the regulation of VEGF, thereby inducing angiogenesis $(\alpha \mathrm{V}, \beta 3$ and $\beta 5$ ) in endothelial cells (40). We, therefore, hypothesized 
that MMP-2/MMP-9 and VEGF are central mediators in CRC invasion and metastasis.

Although accumulating evidence has shown that miR-9 is upregulated in several cancers (41-44), in contrast, many studies have shown downregulation of miR-9 in cervical adenocarcinoma, gastric, ovarian and hepatocellular carcinoma (20-24), indicating a diverse role of miR-9 in different cancer types. These results are studies with a small number of patients. In this study, miR-9 expression level in 60 paired CRC specimens was significantly upregulated in normal tissues compared with paired tumor tissues. Moreover, our data showed that level miR-9 expression inversely correlates with the level of TM4SF1 expression in the same CRC tumor specimens. These findings suggest that miR-9 expression is an important factor in the initiation and early stages of the development of CRC.

We could not determine a survival correlation between miR-9 and TM4SF1 in CRC patients, because survival information was not provided by Biobank of Korea. However, Shiedeck et al (45) have reported that overall 3-year survival is significantly lower in patients with L6-positive blood serum compared to patients L6-negative. Further studies are warranted to identify the roles of miR-9 and TM4SF1 in reducing invasion and metastasis in vivo.

In conclusion, miR-9 is downregulated in CRC specimens and plays a crucial role in CRC invasion and metastasis through regulation of TM4SF1 expression. Our results provide information on a novel mechanism through which miR-9 inhibits the invasion and metastasis of CRC through inhibiting TM4SF1 expression regulation of MMP-2/MMP-9/VEGF in vitro (Fig. 7). Collectively, these findings not only help us understand the molecular mechanism for CRC invasion and metastasis, but also provide a strong rationale to further investigate miR-9 as a potential biomarker for CRC.

\section{Acknowledgements}

The biospecimens and data used in the present study were provided by the Biobank of Chonbuk National University Hospital, a member of the Korea Biobank Network, which is supported by the Ministry of Health, Welfare and Family Affairs. All samples derived from the Korea Biobank Network were obtained with informed consent under institutional review board-approved protocols. This research was supported by the Basic Science Research Program through the National Research Foundation of Korea (NRF), funded by the Ministry of Science, ICT, and the Future Planning (NRF-2015R1D1A3A01016026) and by the Research Institute of Clinical Medicine of Chonbuk National University Hospital-Biomedical Research Institute of Chonbuk National University Hospital.

\section{References}

1. Siegel R, Ma J, Zou Z and Jemal A: Cancer statistics, 2014. CA Cancer J Clin 64: 9-29, 2014

2. O'Connell MJ, Campbell ME, Goldberg RM, Grothey A, Seitz JF, Benedetti JK, André T, Haller DG and Sargent DJ: Survival following recurrence in stage II and III colon cancer: Findings from the ACCENT data set. J Clin Oncol 26: 2336-2341, 2008.
3. Hellstrom I, Horn D, Linsley P, Brown JP, Brankovan V, Hellstrom KE: Monoclonal mouse antibodies raised against human lung carcinoma. Cancer Res 46: 3917-3923, 1986.

4. O'Donnell RT, DeNardo SJ, Shi XB, Mirick GR, DeNardo GL, Kroger LA and Meyers FJ: L6 monoclonal antibody binds prostate cancer. Prostate 37: 91-97, 1998.

5. Wright MD, Ni J and Rudy GB: The L6 membrane proteins - a new four-transmembrane superfamily. Protein Sci 9: 1594-1600, 2000.

6. Chang YW, Chen SC, Cheng EC, Ko YP, Lin YC, Kao YR, Tsay YG, Yang PC, Wu CW and Roffler SR: CD13 (aminopeptidase N) can associate with tumor-associated antigen L6 and enhance the motility of human lung cancer cells. Int J Cancer 116: 243-252, 2005.

7. Lekishvili T, Fromm E, Mujoomdar M and Berditchevski F: The tumour-associated antigen L6 (L6-Ag) is recruited to the tetraspanin-enriched microdomains: Implication for tumour cell motility. J Cell Sci 121: 685-694, 2008.

8. Kao YR, Shih JY, Wen WC, Ko YP, Chen BM, Chan YL, Chu YW, Yang PC, Wu CW and Roffler SR: Tumor-associated antigen L6 and the invasion of human lung cancer cells. Clin Cancer Res 9: 2807-2816, 2003.

9. Marken JS, Schieven GL, Hellström I, Hellström KE and Aruffo A: Cloning and expression of the tumor-associated antigen L6. Proc Natl Acad Sci USA 89: 3503-3507, 1992.

10. Allioli N, Vincent S, Vlaeminck-Guillem V, DecaussinPetrucci M, Ragage F, Ruffion A and Samarut J: TM4SF1, a novel primary androgen receptor target gene over-expressed in human prostate cancer and involved in cell migration. Prostate 71: 1239-1250, 2011.

11. Otsuka M, Kato M, Yoshikawa T, Chen H, Brown EJ, Masuho Y, Omata M and Seki N: Differential expression of the L-plastin gene in human colorectal cancer progression and metastasis. Biochem Biophys Res Commun 289: 876-881, 2001.

12. Ying SY, Chang DC, Miller JD and Lin SL: The microRNA: Overview of the RNA gene that modulates gene functions. Methods Mol Biol 342: 1-18, 2006.

13. Wang F, Xue X, Wei J, An Y, Yao J, Cai H, Wu J, Dai C, Qian Z, $\mathrm{Xu} \mathrm{Z}$, et al: hsa-miR-520h downregulates ABCG2 in pancreatic cancer cells to inhibit migration, invasion, and side populations. Br J Cancer 103: 567-574, 2010.

14. Zhou L, Liu F, Wang X and Ouyang G: The roles of microRNAs in the regulation of tumor metastasis. Cell Biosci 5: 32, 2015.

15. Aguda BD, Kim Y,Piper-Hunter MG, Friedman A and Marsh CB: MicroRNA regulation of a cancer network: Consequences of the feedback loops involving miR-17-92, E2F, and Myc. Proc Natl Acad Sci USA 105: 19678-19683, 2008.

16. Grady WM, Parkin RK, Mitchell PS, Lee JH, Kim YH, Tsuchiya KD, Washington MK, Paraskeva C, Willson JK, $\mathrm{Kaz} \mathrm{AM}$, et al: Epigenetic silencing of the intronic microRNA hsa-miR-342 and its host gene EVL in colorectal cancer. Oncogene 27: 3880-3888, 2008.

17. Mo JS, Alam KJ, Kang IH, Park WC, Seo GS, Choi SC, Kim HS, Moon HB, Yun KJ and Chae SC: MicroRNA 196B regulates FAS-mediated apoptosis in colorectal cancer cells. Oncotarget 6: 2843-2855, 2015 .

18. Hoffman AE, Zheng T, Yi C, Leaderer D, Weidhaas J, Slack F, Zhang Y, Paranjape T and Zhu Y: microRNA miR-196a-2 and breast cancer: A genetic and epigenetic association study and functional analysis. Cancer Res 69: 5970-5977, 2009.

19. Aqeilan RI, Calin GA and Croce CM: miR-15a and miR-16-1 in cancer: Discovery, function and future perspectives. Cell Death Differ 17: 215-220, 2010.

20. Zhang J, Jia J, Zhao L, Li X, Xie Q, Chen X, Wang J and Lu F: Down-regulation of microRNA-9 leads to activation of IL-6/Jak/ STAT3 pathway through directly targeting IL-6 in HeLa cell. Mol Carcinog: Mar 25, 2015. n/a, 2015. doi: 10.1002/mc.22317. (Epub ahead of print)

21. Mohammadi-Yeganeh S, Mansouri A and Paryan M: Targeting of miR9/NOTCH1 interaction reduces metastatic behavior in triplenegative breast cancer. Chem Biol Drug Des 86: 1185-1191, 2015.

22. Laios A, O'Toole S, Flavin R, Martin C, Kelly L, Ring M, Finn SP, Barrett C, Loda M, Gleeson N, et al: Potential role of miR-9 and miR-223 in recurrent ovarian cancer. Mol Cancer 7: 35, 2008.

23. Higashi T, Hayashi H, Ishimoto T, Takeyama H, Kaida T, Arima K, Taki K, Sakamoto K, Kuroki H, Okabe H, et al: miR-9-3p plays a tumour-suppressor role by targeting TAZ (WWTR1) in hepatocellular carcinoma cells. Br J Cancer 113: 252-258, 2015. 
24. Luo H, Zhang H, Zhang Z, Zhang X, Ning B, Guo J, Nie N, Liu B and Wu X: Down-regulated miR-9 and miR-433 in human gastric carcinoma. J Exp Clin Cancer Res 28: 82, 2009.

25. Tagawa T, Haraguchi T, Hiramatsu H, Kobayashi K, Sakurai K, Inada $\mathrm{K}$ and Iba $\mathrm{H}$ : Multiple microRNAs induced by $\mathrm{Cdx} 1$ suppress $\mathrm{Cdx} 2$ in human colorectal tumour cells. Biochem J 447: 449-455, 2012

26. Zhu M, Xu Y, Ge M, Gui Z and Yan F: Regulation of UHRF1 by microRNA-9 modulates colorectal cancer cell proliferation and apoptosis. Cancer Sci 106: 833-839, 2015.

27. Sarver AL, French AJ, Borralho PM, Thayanithy V, Oberg AL, Silverstein KA, Morlan BW, Riska SM, Boardman LA, Cunningham JM, et al: Human colon cancer profiles show differential microRNA expression depending on mismatch repair status and are characteristic of undifferentiated proliferative states. BMC Cancer 9: 401, 2009.

28. Slattery ML, Herrick JS, Mullany LE, Valeri N, Stevens J, Caan BJ, et al: An evaluation and replication of miRNAs with disease stage and colorectal cancer-specific mortality. Int J Cancer 137: 428-438, 2015.

29. Bandres E, Agirre X, Bitarte N, Ramirez N, Zarate R, RomanGomez J, Prosper F and Garcia-Foncillas J: Epigenetic regulation of microRNA expression in colorectal cancer. Int J Cancer 125: 2737-2743, 2009.

30. Rotkrua P, Akiyama Y, Hashimoto Y, Otsubo T and Yuasa Y: MiR-9 downregulates CDX2 expression in gastric cancer cells. Int J Cancer 129: 2611-2620, 2011.

31. Kim SL, Liu YC, Park YR, Seo SY, Kim SH, Kim IH, Lee SO, Lee ST, Kim DG and Kim SW: Parthenolide enhances sensitivity of colorectal cancer cells to TRAIL by inducing death receptor 5 and promotes TRAIL-induced apoptosis. Int $\mathrm{J}$ Oncol 46 : 1121-1130, 2015

32. Liu LP, Liang HF, Chen XP, Zhang WG, Yang SL, Xu T and Ren L: The role of NF-kappaB in Hepatitis $b$ virus $X$ proteinmediated upregulation of VEGF and MMPs. Cancer Invest 28 443-451, 2010.

33. Kim Y, Kang H, Jang SW and Ko J: Celastrol inhibits breast cancer cell invasion via suppression of NF- $\kappa \mathrm{B}$-mediated matrix metalloproteinase-9 expression. Cell Physiol Biochem 28: $175-184,2011$

34. Lu J, Getz G, Miska EA, Alvarez-Saavedra E, Lamb J, Peck D, Sweet-Cordero A, Ebert BL, Mak RH, Ferrando AA, et al: MicroRNA expression profiles classify human cancers. Nature 435: 834-838, 2005
35. Landgraf P, Rusu M, Sheridan R, Sewer A, Iovino N, Aravin A, Pfeffer S, Rice A, Kamphorst AO, Landthaler M, et al: A mammalian microRNA expression atlas based on small RNA library sequencing. Cell 129: 1401-1414, 2007.

36. Duffy MJ, Maguire TM, Hill A, McDermott E and O'Higgins N: Metalloproteinases: Role in breast carcinogenesis, invasion and metastasis. Breast Cancer Res 2: 252-257, 2000.

37. Hanemaaijer R, Verheijen JH, Maguire TM, Visser H, Toet K, McDermott E, O'Higgins N and Duffy MJ: Increased gelatinase$\mathrm{A}$ and gelatinase-B activities in malignant vs. benign breast tumors. Int J Cancer 86: 204-207, 2000.

38. Chambers AF and Matrisian LM: Changing views of the role of matrix metalloproteinases in metastasis. J Natl Cancer Inst 89: 1260-1270, 1997.

39. Mc Donnell S, Chaudhry V, Mansilla-Soto J, Zeng ZS, Shu WP and Guillem JG: Metastatic and non-metastatic colorectal cancer (CRC) cells induce host metalloproteinase production in vivo. Clin Exp Metastasis 17: 341-349, 1999.

40. Shih SC, Zukauskas A, Li D, Liu G, Ang LH, Nagy JA, Brown LF and Dvorak HF: The L6 protein TM4SF1 is critical for endothelial cell function and tumor angiogenesis. Cancer Res 69: 3272-3277, 2009.

41. Nass D, Rosenwald S, Meiri E, Gilad S, Tabibian-Keissar H, Schlosberg A, Kuker H, Sion-Vardy N, Tobar A, Kharenko O, et al: MiR-92b and miR-9/9* are specifically expressed in brain primary tumors and can be used to differentiate primary from metastatic brain tumors. Brain Pathol 19: 375-383, 2009.

42. Ma L, Young J, Prabhala H, Pan E, Mestdagh P, Muth D, TeruyaFeldstein J, Reinhardt F, Onder TT, Valastyan S, et al: miR-9, a MYC/MYCN-activated microRNA, regulates E-cadherin and cancer metastasis. Nat Cell Biol 12: 247-256, 2010.

43. Shiiyama R, Fukushima S, Jinnin M, Yamashita J, Miyashita A, Nakahara S, Kogi A, Aoi J, Masuguchi S, Inoue Y, et al: Sensitive detection of melanoma metastasis using circulating microRNA expression profiles. Melanoma Res 23: 366-372, 2013.

44. Song Y, Li J, Zhu Y, Dai Y, Zeng T, Liu L, Li J, Wang H, Qin Y, Zeng M, et al: MicroRNA-9 promotes tumor metastasis via repressing E-cadherin in esophageal squamous cell carcinoma. Oncotarget 5: 11669-11680, 2014. 